\title{
Grid-Connected Voltage Source Converters with integrated Multilevel-Based Active Filters
}

\author{
Daniel Bernet, Lukas Stefanski, Rüdiger Schwendemann, Christoph Rollbühler, Marc Hiller \\ Karlsruhe Institute of Technology, \\ Institute of Electrical Engineering (ETI) \\ Kaiserstr. 12, 76131 Karlsruhe, Germany \\ daniel.bernet@kit.edu
}

\begin{abstract}
Low- and medium voltage power converters for grid applications require bulky and expensive LCL-filters in order to meet the harmonic limits defined by the grid code. This paper presents a novel hybrid converter topology with smaller passive components, composed of a high power main converter with an integrated low power active filter. The presented coupled control strategies for a current-source and a voltage-source topology enable the immediate mitigation of the switching frequency current ripple, significantly decreasing the total harmonic distortion (THD) of the output current to below $0.5 \%$ and thereby eliminating the need for a passive LCL-filter. Compared to LCL-filter based converter systems, a reduction of the inductive component expenditure of up to $50 \%$ is achievable even for a very low active filter to main converter power rating ratio of $10 \%$. The outstanding harmonic performance and reduced passive component demand of the hybrid converter allow increased power density and efficiency as well as lower costs of low- and medium voltage power converters.
\end{abstract}

Index Terms-Hybrid converter systems, active filter (AF), multilevel converter, control systems, LCL filter, active front end

\section{INTRODUCTION}

Grid-connected power converters are widely used in applications such as the integration of renewable energies (e.g. photovoltaics and wind) in electrical grids, charging stations for electric vehicles and a large number of electric loads such as industrial electric drives. The main requirements for grid converters are high efficiency, low costs, high reliability and high power density while at the same time meeting the harmonic limits as defined by the grid code. Nine out of ten manufacturers offer the widely distributed Two-Level power converter as machine- and grid-side converter solution in the low voltage range up to $1 \mathrm{kV}$, as determined by a market analysis. The converters are generally operated at switching frequencies between $3 \mathrm{kHz}$ and $20 \mathrm{kHz}$. In the lower medium voltage range from $2.3 \mathrm{kV}$ to $4.16 \mathrm{kV}$, half of the commercially available converter solutions use the 3L-Neutral Point Clamped (NPC) converter. The $3 \mathrm{~L}-\mathrm{NPC}$ is typically operated at a switching frequency range of several hundred Hertz $(200 \mathrm{~Hz}$ to $600 \mathrm{~Hz})$ per switch. Due to the low number of voltage levels and the limited switching frequency, power converters in the low- and

The authors would like to thank the German Federal Ministry of Education and Research for funding this research within the ENSURE project under the grant FKZ 03SFK1A.

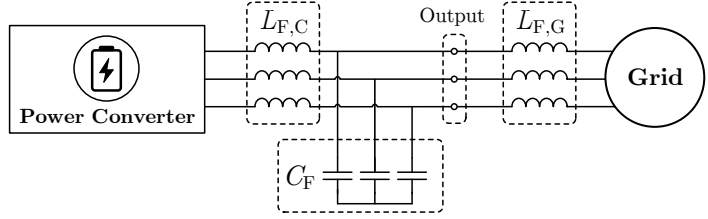

Fig. 1. Conventional LCL-filter based grid-connected converter system

medium voltage range require passive output filters in order to meet harmonic limits, e.g. given by the IEEE Standard 519. Therefore, passive LCL-filters composed of bulky inductors and capacitors are commonly used in the converter systems as shown in Fig. 1, often entailing costs that surpass the costs of the power converter. Further disadvantages of passive LCL filters are substantial additional losses, a reduced dynamic performance, large volume and size as well as frequency dependency and oscillation susceptibility, requiring significant and lossy attenuation.

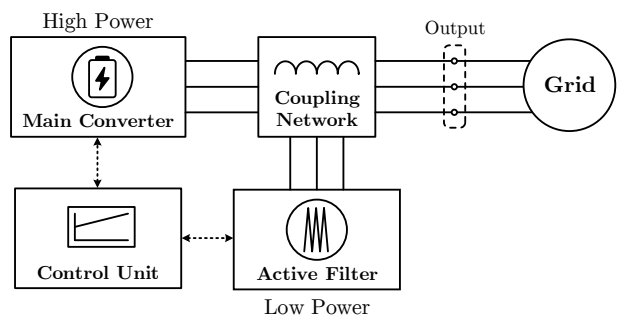

Fig. 2. Proposed hybrid converter topology composed of a high power main converter (MC) and a low power active filter (AF)

In order to overcome the aforementioned disadvantages, this work focuses on the integration of new active filter topologies into grid-connected power converters and thereby eliminating the need for a passive LCL-filter. Fig. 2 shows the proposed hybrid converter system, composed of a high power main converter (MC), a low power active filter (AF) and a single control unit. Due to the wide range of applications as a result of competitive costs and high robustness, Two- and ThreeLevel converters are most suitable for the application as main converter within the hybrid converter system. Although any Voltage Source Converter (VSC) topology can be used as active filter in principle, it will be seen that the application of Multilevel-based active filters is most suitable for attaining a 
superior output power quality of the hybrid converter. The main converter operates as a conventional grid-connected converter, enabling the exchange of fundamental active and reactive power. By replacing the output LCL-filter, the active filter mitigates the switching frequency current ripple of the main converter and thereby eliminates the oscillation susceptibility and supersedes the lossy attenuation.

\section{PARt I: Current-Source Hybrid Converter}

In previous works, systems with integrated active filters have been investigated e.g. for a diode front end [1] and a singlephase onboard charger for electric vehicles [2]. Moreover, control strategies of grid-connected power converters allowing both the control of fundamental power and compensation of harmonics have been implemented [3]. However, these control strategies were based on a current source operation, allowing the elimination of distinct harmonic frequencies. In comparison, the objective of the proposed hybrid converter is the mitigation of the entire switching frequency current ripple with a coupled control of the main converter and the integrated active filter.

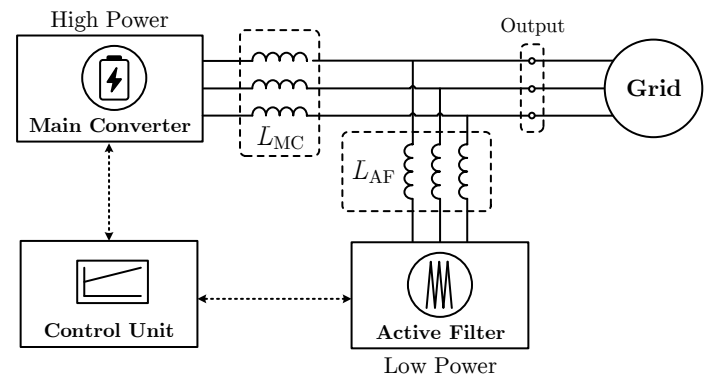

Fig. 3. Proposed grid-connected current-source hybrid converter topology

Unlike conventional active filter applications, the main converter, the active filter, the control unit and the coupling network constitute the proposed single converter system, allowing a coupled control of main converter and active filter. As shown in Fig. 3, a coupling network commonly used for active filter applications is considered first, consisting of main converter and active filter output inductances and resulting in a current-source output characteristic of the hybrid converter.

\section{A. Converter Topology and Analysis}

In this paper, the proposed hybrid converter is composed of a Two-Level Voltage Source Converter (2L-VSC) combined with an active filter made up of cascaded full-bridge cells in star configuration (Fig. 4). The topology used for the active filter is also known as Cascaded H-Bridge (CHB) STATCOM [4] or Modular Multilevel STATCOM in single-star configuration [5] and, in delta configuration, as SVC PLUS by SIEMENS [6] or SVC Light by ABв [7].

The three-phase output voltage of the $2 \mathrm{~L}-\mathrm{VSC} \boldsymbol{u}_{\mathrm{MC}}=$ $\left[\begin{array}{lll}u_{\mathrm{MC}, \mathrm{u}} & u_{\mathrm{MC}, \mathrm{v}} & u_{\mathrm{MC}, \mathrm{w}}\end{array}\right]^{\mathrm{T}}$ is composed of the fundamental voltage ${ }^{1} \boldsymbol{u}_{\mathrm{MC}}=\left[{ }^{1} u_{\mathrm{MC}, \mathrm{u}}{ }^{1} u_{\mathrm{MC}, \mathrm{v}}{ }^{1} u_{\mathrm{MC}, \mathrm{w}}\right]^{\mathrm{T}}$ and a ripple voltage $\sum \nu \boldsymbol{u}_{\mathrm{MC}}=\left[{ }^{\sum \nu} u_{\mathrm{MC}, \mathrm{u}} \sum^{\sum \nu} u_{\mathrm{MC}, \mathrm{v}} \sum^{\sum \nu} u_{\mathrm{MC}, \mathrm{w}}\right]^{\mathrm{T}}$, according to

$$
\boldsymbol{u}_{\mathrm{MC}}={ }^{1} \boldsymbol{u}_{\mathrm{MC}}+{ }^{\sum \nu} \boldsymbol{u}_{\mathrm{MC}}
$$

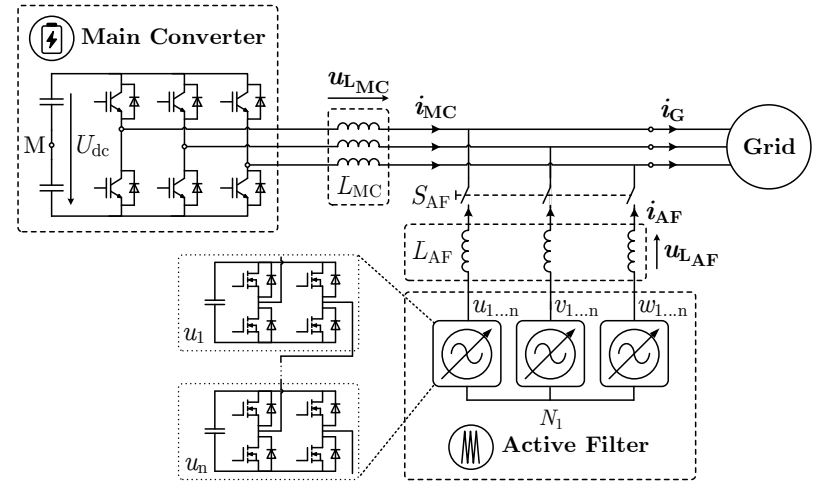

Fig. 4. Proposed current-source hybrid converter composed of a $2 \mathrm{~L}-\mathrm{VSC}$ as main converter and a CHB STATCOM in star configuration as active filter

where $\nu=\{h \in \mathbb{N} \mid h \geq 2\}$. Due to the grid connection, the output current $\boldsymbol{i}_{\mathrm{MC}}=\left[\begin{array}{ll}i_{\mathrm{MC}, \mathrm{u}} & i_{\mathrm{MC}, \mathrm{v}} i_{\mathrm{MC}, \mathrm{w}}\end{array}\right]^{\mathrm{T}}$ of the $2 \mathrm{~L}-\mathrm{VSC}$ results from the voltage drop across the coupling inductance $L_{\mathrm{MC}}$ with the winding resistance $R_{\mathrm{MC}}$, given by

$$
\begin{aligned}
\boldsymbol{u}_{\mathbf{L}_{\mathrm{MC}}} & =\left[\begin{array}{lll}
u_{\mathrm{L}_{\mathrm{MC}}, \mathrm{u}} & u_{\mathrm{L}_{\mathrm{MC}}, \mathrm{v}} & u_{\mathrm{L}_{\mathrm{MC}}, \mathrm{w}}
\end{array}\right]^{\mathrm{T}}=\boldsymbol{u}_{\mathrm{MC}}-\boldsymbol{u}_{\mathrm{G}} \\
& =R_{\mathrm{MC}} \boldsymbol{i}_{\mathrm{MC}}+L_{\mathrm{MC}} \frac{\mathrm{d} \boldsymbol{i}_{\mathrm{MC}}}{\mathrm{d} t}
\end{aligned}
$$

where $u_{\mathrm{G}}=\left[\begin{array}{lll}u_{\mathrm{G}, \mathrm{u}} & u_{\mathrm{G}, \mathrm{v}} & u_{\mathrm{G}, \mathrm{w}}\end{array}\right]^{\mathrm{T}}$ denotes the grid voltage. It is assumed that the grid voltage is an undistorted fundamental voltage $\boldsymbol{u}_{\mathrm{G}}={ }^{1} \boldsymbol{u}_{\mathrm{G}}=\left[{ }^{1} u_{\mathrm{G}, \mathrm{u}}{ }^{1} u_{\mathrm{G}, \mathrm{v}}{ }^{1} u_{\mathrm{G}, \mathrm{w}}\right]^{\mathrm{T}}$, resulting in

$$
\boldsymbol{u}_{\mathrm{L}_{\mathrm{MC}}}={ }^{\mathbf{1}} \boldsymbol{u}_{\mathrm{MC}}-{ }^{\mathbf{1}} \boldsymbol{u}_{\mathrm{G}}+{ }^{\sum \nu} \boldsymbol{u}_{\mathrm{MC}}
$$

where ${ }^{\mathbf{1}} \boldsymbol{u}_{\mathrm{MC}}-{ }^{\mathbf{1}} \boldsymbol{u}_{\mathrm{G}}$ corresponds to the fundamental voltage drop and $\sum \nu \boldsymbol{u}_{\mathrm{MC}}$ to the ripple voltage drop. Based on the ratio $\omega L_{\mathrm{MC}}>>R_{\mathrm{MC}}$, the winding resistance of the coupling inductor will be neglected in the following. Consequently, (2) can be rewritten as

$$
\begin{aligned}
& \boldsymbol{i}_{\mathrm{MC}}=\underbrace{\frac{1}{L_{\mathrm{MC}}} \int\left({ }^{\mathbf{1}} \boldsymbol{u}_{\mathrm{MC}}-{ }^{\mathbf{1}} \boldsymbol{u}_{\mathrm{G}}\right) \mathrm{d} t}_{{ }^{1} \boldsymbol{i}_{\mathrm{MC}}=\left[{ }^{1} i_{\mathrm{MC}, \mathrm{u}}{ }^{1} i_{\mathrm{MC}, \mathrm{v}}{ }^{1} i_{\mathrm{MC}, \mathrm{w}}\right]^{\mathrm{T}}} \\
& +\underbrace{\frac{1}{L_{\mathrm{MC}}} \int \sum \boldsymbol{\nu} \boldsymbol{u}_{\mathrm{MC}} \mathrm{d} t}_{\sum \boldsymbol{\nu}_{\boldsymbol{M} \mathbf{C}}=\left[\sum \nu i_{\mathrm{MC}, \mathrm{u}} \sum \nu i_{\mathrm{MC}, \mathrm{v}} \sum \nu i_{\mathrm{MC}, \mathrm{w}}\right]^{\mathrm{T}}}
\end{aligned}
$$

As expected, the fundamental portion of the main converter voltage leads to a fundamental current injection ${ }^{1} i_{\mathrm{MC}}$ into the grid, while the ripple voltage causes an injected ripple current $\sum \nu i_{\text {MC }}$. Kirchoff's Current Law at the converter output yields

$$
\boldsymbol{i}_{\mathrm{G}}={ }^{1} \boldsymbol{i}_{\mathrm{G}}+{ }^{\sum \nu} \boldsymbol{i}_{\mathrm{G}}={ }^{1} \boldsymbol{i}_{\mathrm{MC}}+{ }^{\sum \nu} \boldsymbol{i}_{\mathrm{MC}}+\boldsymbol{i}_{\mathrm{AF}}
$$

where $\boldsymbol{i}_{\mathrm{AF}}=\left[\begin{array}{lll}i_{\mathrm{AF}, \mathrm{u}} & i_{\mathrm{AF}, \mathrm{v}} & i_{\mathrm{AF}, \mathrm{w}}\end{array}\right]^{\mathrm{T}}$ is the current of the CHB STATCOM. Since the grid ripple current $\sum \boldsymbol{\nu}_{\mathrm{G}}=$ $\left[\sum \nu i_{\mathrm{G}, \mathrm{u}} \sum \nu i_{\mathrm{G}, \mathrm{v}} \sum \nu i_{\mathrm{G}, \mathrm{w}}\right]^{\mathrm{T}}$ has to meet harmonic limits given by the grid code, conventional grid-connected power converters use an expensive and bulky LCL-Filter at the converter output. For the proposed hybrid converter, this can be achieved by the injection of an inverse ripple current using the CHB STATCOM, as can be seen in (5). This can be 
accomplished by applying a voltage drop across the coupling inductance $L_{\mathrm{AF}}$ that leads to an inversely phased ripple current compared to the ripple current passing through $L_{\mathrm{MC}}$. According to (2) it follows that

$$
\begin{aligned}
\frac{\mathrm{d}^{\sum} \boldsymbol{\nu} \boldsymbol{i}_{\mathrm{AF}}}{\mathrm{d} t}=\frac{\sum \boldsymbol{\nu} \boldsymbol{u}_{\mathbf{L A F}}}{L_{\mathrm{AF}}}= & -\frac{\sum \boldsymbol{\nu} \boldsymbol{u}_{\mathrm{MC}}}{L_{\mathrm{MC}}}=-\frac{\mathrm{d}^{\sum} \boldsymbol{\nu} \boldsymbol{i}_{\mathrm{MC}}}{\mathrm{d} t} \\
\sum \boldsymbol{\nu} \boldsymbol{u}_{\mathbf{L}_{\mathrm{AF}}} & =-\frac{L_{\mathrm{AF}}}{L_{\mathrm{MC}}} \boldsymbol{\nu}_{\boldsymbol{u}_{\mathbf{M C}}}
\end{aligned}
$$

where $\sum \nu i_{\mathrm{AF}}=\left[\sum \nu i_{\mathrm{AF}, \mathrm{u}} \sum \nu i_{\mathrm{AF}, \mathrm{v}} \sum \nu i_{\mathrm{AF}, \mathrm{w}}\right]^{\mathrm{T}}$ is the ripple current injected by the CHB STATCOM and $\sum \boldsymbol{\nu} \boldsymbol{u}_{\mathrm{L}_{\mathrm{AF}}}=$ $\left[{ }^{\sum \nu} u_{\mathrm{L}_{\mathrm{AF}}, \mathrm{u}} \sum^{\sum \nu} u_{\mathrm{L}_{\mathrm{AF}}, \mathrm{v}} \sum^{\sum \nu} u_{\mathrm{L}_{\mathrm{AF}}, \mathrm{w}}\right]^{\mathrm{T}}$ the ripple voltage drop across the coupling inductance $L_{\mathrm{AF}}$. Kirchhoff's Voltage Law yields the reference voltage of the CHB STATCOM in (7).

$$
\begin{aligned}
\boldsymbol{u}_{\mathrm{AF}}^{*} & =\boldsymbol{u}_{\mathrm{G}}+{ }^{\sum \nu} \boldsymbol{u}_{\mathbf{L}_{\mathrm{AF}}} \\
& =\boldsymbol{u}_{\mathrm{G}}-\frac{L_{\mathrm{AF}} \sum \boldsymbol{\nu}}{L_{\mathrm{MC}}} \boldsymbol{u}_{\mathrm{MC}} \\
& =\boldsymbol{u}_{\mathrm{G}}-\frac{L_{\mathrm{AF}}}{L_{\mathrm{MC}}}\left(\boldsymbol{u}_{\mathrm{MC}}-{ }^{\mathbf{1}} \boldsymbol{u}_{\mathrm{MC}}\right)
\end{aligned}
$$

Thus, an elimination of the switching frequency harmonics of the power converter output current can be achieved by applying the reference voltage $\boldsymbol{u}_{\mathrm{AF}}^{*}$ to the CHB STATCOM. Since both $\boldsymbol{u}_{\mathrm{G}}$ and ${ }^{1} \boldsymbol{u}_{\mathrm{MC}}$ are sinusoidal voltages, a multilevel output voltage $\boldsymbol{u}_{\mathrm{AF}}$ with a high number of voltage levels is most suitable for the CHB STATCOM in order to ensure an accurate switching frequency current ripple mitigation.

\section{B. Converter Control}

The designed control scheme of the hybrid converter is shown in Fig. 5. As can be seen in (7), the reference voltage of the CHB STATCOM is dependent on the output voltage of the Two-Level converter, requiring a coupled control of the main converter and the active filter. The high power TwoLevel converter is operated with a conventional grid-side control using Space Vector Modulation (SVM). The reference currents $i_{\mathrm{G}, \mathrm{dq}}^{*}=i_{\mathrm{G}, \mathrm{d}}^{*}+j i_{\mathrm{G}, \mathrm{q}}^{*}$ are determined by the application dependent reference values for the exchange of active and reactive power. According to (7), the reference voltage $\boldsymbol{u}_{\mathrm{AF}}^{*}$ of the CHB STATCOM is calculated using the grid voltage $\boldsymbol{u}_{\mathrm{G}}$, the pulsed output voltage $\boldsymbol{u}_{\mathrm{MC}}$ of the Two-Level converter and its fundamental voltage ${ }^{1} \boldsymbol{u}_{\mathrm{MC}}$. In order to avoid the measurement of $\boldsymbol{u}_{\mathrm{MC}}$, the pulsed voltage is recreated using the

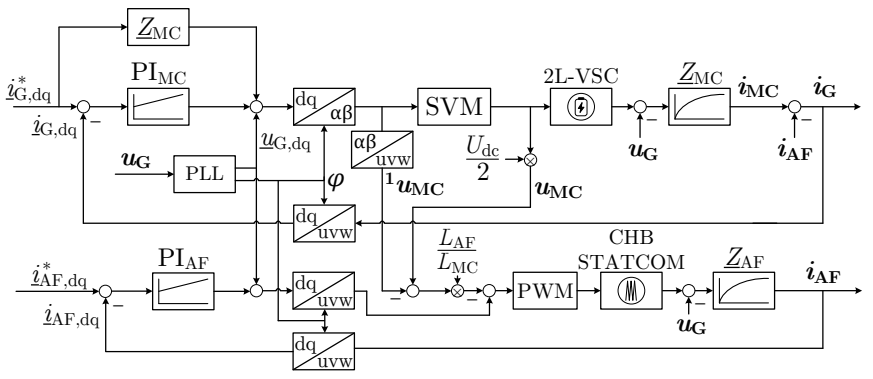

Fig. 5. Control scheme of the proposed current-source hybrid converter switching pattern generated by the SVM and the measured DClink voltage $U_{\mathrm{dc}}$. Since the control of the Two-Level converter is a fundamental frequency control scheme, the fundamental voltage ${ }^{1} \boldsymbol{u}_{\mathrm{MC}}$ corresponds to the output of the main converters current control loop. To ensure a high operating stability of the hybrid converter system, an additional control loop of the fundamental current ${ }^{1} i_{\mathrm{AF}}$ is applied, where the reference currents $\underline{i}_{\mathrm{AF}, \mathrm{dq}}^{*}$ could result from a superordinate control based on [8], performing an accurate energy balancing of the CHB STATCOM.

For the practical implementation, parameter sensitivity and the recreation of the Two-Level converter output voltage $\boldsymbol{u}_{\mathrm{MC}}$ are possible causes of control deviations. Therefore, the applied locking times and, as required, the non-ideal switching behaviour of the power semiconductors has to be taken into account for the voltage recreation. Nevertheless, the challenge of voltage calculation using the switching pattern has been solved in previous works for applications with high demands on accuracy, e.g. for a flux observer applied in the model predictive control of an induction motor [9].

\section{Simulation Results}

Simulation results are carried out for the parameters given in Tab. I. In a first step, identical coupling inductances $L_{\mathrm{MC}}=L_{\mathrm{AF}}$ as well as fed DC-links of the full bridge cells are used for the investigation. Since the CHB STATCOM merely provides the reactive power of the harmonic distortion, its rated power is distinctly lower compared to the Two-Level converter. Therefore, the switching frequency of the CHB STATCOM is set to $50 \mathrm{kHz}$, yielding in a high output voltage quality.

Fig. 6 shows the simulation results for the line-to-line voltages, the main converter and active filter output currents as well as the injected grid current. The grid-connected hybrid converter is at first operated at no load and without the active filter. At $t=50 \mathrm{~ms}$, the grid current reference changes to $i_{\mathrm{G}, \mathrm{d}}^{*}=200 \mathrm{~A}$ and at $t=100 \mathrm{~ms}$ the active filter is connected to the converter output by closing the switch $S_{\mathrm{AF}}$ (see Fig. 4). In order to demonstrate the dynamic behavior of the switching frequency current ripple mitigation, the reference current changes to $400 \mathrm{~A}$ at $t=150 \mathrm{~ms}$.

As mentioned in Section II-A, the reference voltage of the active filter $\boldsymbol{u}_{\mathrm{AF}}^{*}$ is to be approximated by the CHB STATCOM using $n=12$ cells per phase and 25 voltage levels. Due to the equal coupling inductances, the maxima of $\boldsymbol{u}_{\mathrm{AF}}^{*}$ are according to (7) considerably higher than the grid voltage $\boldsymbol{u}_{\mathrm{G}}$. In consequence of the high ripple current, the grid voltages are distorted before $t=100 \mathrm{~ms}$. After the connection of the CHB STATCOM the switching frequency current ripple is almost entirely compensated, leading to an improved quality of the injected grid current and, moreover, to an improved grid voltage quality. Fig. 6 shows very short rise times of the main converter and grid currents at $t=50 \mathrm{~ms}$ and $t=150 \mathrm{~ms}$, respectively, and thereby reveals an excellent dynamic behavior of the hybrid converter. Despite the large grid current increase, the amplitudes of the active filter currents remain nearly unaffected. 
TABLE I

SIMULATION PARAMETERS OF THE CURRENT-SOURCE HYBRID CONVERTER

\begin{tabular}{|c|c|c|c|c|c|c|c|c|c|c|}
\hline Grid Volt. & DC-link Volt. & Num. of Cells & Cell Volt. & \multicolumn{2}{c|}{ Switch. Freq. } & \multicolumn{2}{c|}{ Coupling Inductances } & \multicolumn{2}{c|}{ Grid } \\
\hline$U_{\mathrm{G}}$ & $U_{\mathrm{dc}, \mathrm{MC}}$ & $n_{\mathrm{AF}}$ & $u_{\mathrm{c}, \mathrm{AF}}$ & $f_{\mathrm{sw}, \mathrm{MC}}$ & $f_{\mathrm{sw}, \mathrm{AF}}$ & $L_{\mathrm{MC}}=L_{\mathrm{AF}}$ & $R_{\mathrm{MC}}=R_{\mathrm{AF}}$ & $\omega_{\mathrm{G}}$ & $L_{\mathrm{G}}$ & $R_{\mathrm{G}}$ \\
\hline $400 \mathrm{~V}$ & $700 \mathrm{~V}$ & 12 & $100 \mathrm{~V}$ & $3 \mathrm{kHz}$ & $50 \mathrm{kHz}$ & $200 \mu \mathrm{H}$ & $1 \mathrm{~m} \Omega$ & $2 \pi 50 \mathrm{~Hz}$ & $50 \mu \mathrm{H}$ & $1.3 \mathrm{~m} \Omega$ \\
\hline
\end{tabular}
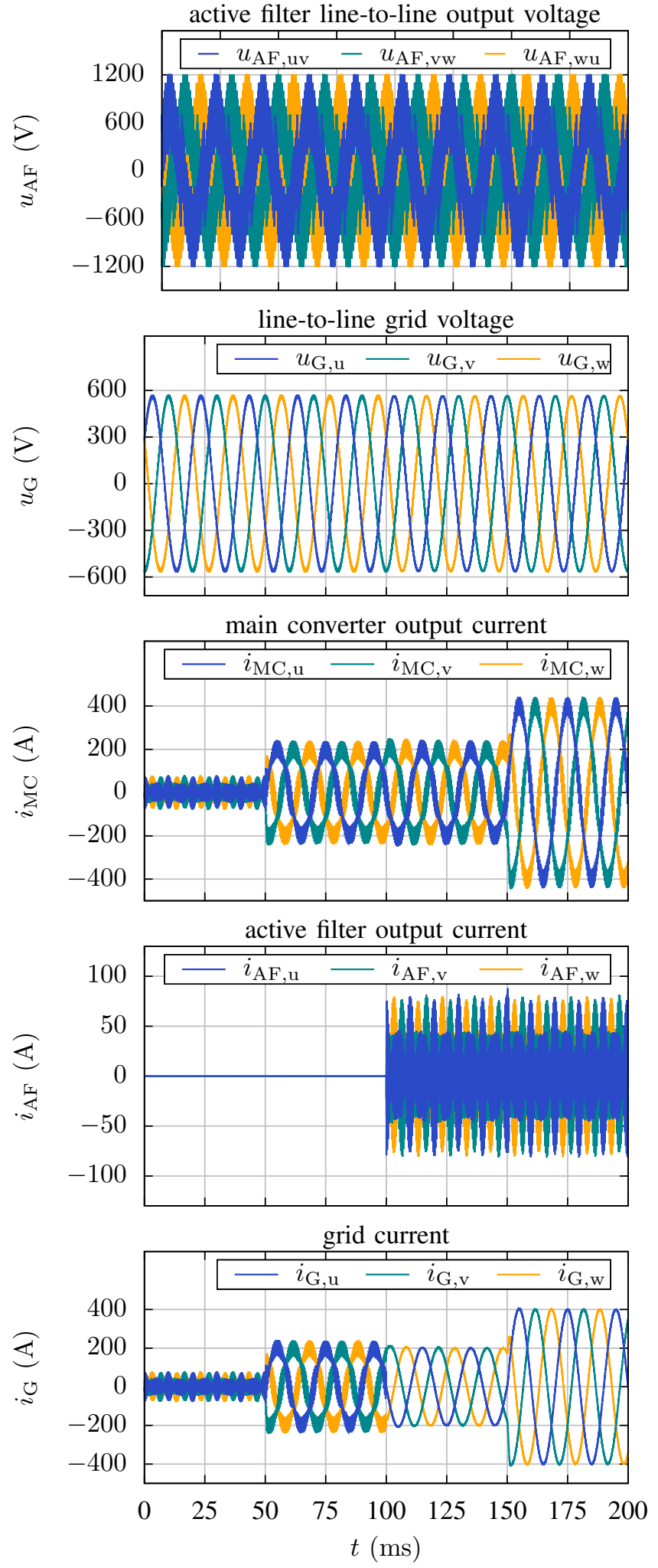

Fig. 6. Simulation results of the current-source hybrid converter
The accurate performance of the hybrid converter is likewise demonstrated by the harmonic spectra depicted in Fig. 7. The harmonic spectrum of the Two-Level converter current during steady state in the time range $100 \mathrm{~ms} \leq t \leq 150 \mathrm{~ms}$, which is composed of the fundamental harmonic current and the expected harmonic frequencies around the switching frequency of $3 \mathrm{kHz}$, has a $\mathrm{THD}_{\mathrm{MC}}$ of $10.2 \%$. The CHB STATCOM current corresponds in good approximation to the harmonic spectrum of the Two-Level converter, whereas the fundamental current is missing and the phase angle of occuring harmonics is shifted by $180^{\circ}$. Thus, the switching frequency current ripple is eliminated and a fundamental grid current with a very low total harmonic distortion of $\mathrm{THD}_{\mathrm{G}}=0.4 \%$ is attained.

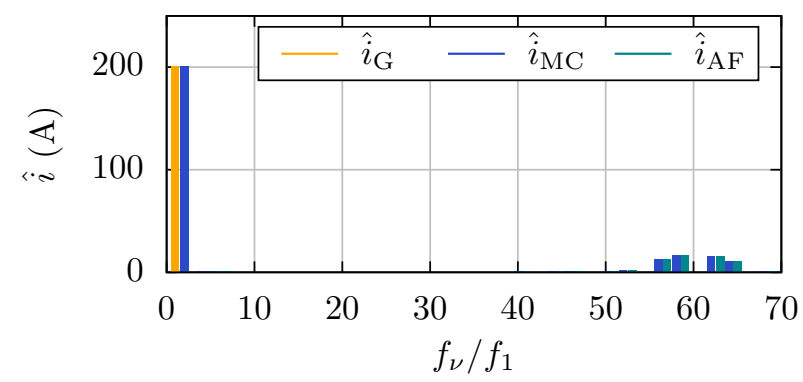

Fig. 7. Harmonic spectra for $i_{\mathrm{G}, \mathrm{d}}^{*}=200 \mathrm{~A}$ with the fundamental frequency $f_{1}=50 \mathrm{~Hz}$ and the harmonic frequency $f_{\nu}=\nu \cdot f_{1}$

\section{Discussion}

The coupled control scheme for the proposed currentsource hybrid converter allows the mitigation of switching frequency current ripple. The simulation results show a superior steady state and dynamic behavior of the hybrid converter under reasonable grid conditions, reducing the total harmonic distortion of the output current to below $0.5 \%$. This excellent harmonic performance reveals a high potential for the reduction of passive component cost compared to LCL-filter based converter systems. Since the integrated active filter solely compensates the ripple current of the main converter for both steady state and dynamic operation, it can be designed for a reduced power rating compared to the main converter. For the practical realization, the dependency on coupling inductance parameters as well as non-ideal converter characteristics due to dead and locking times, non-ideal switching behavior and voltage drops across conducting power semiconductors can be possible causes of performance impairments. A major disadvantage of the investigated converter configuration is the high voltage demand of the active filter. According to (7), a reduction of the active filter reference voltage can be achieved by decreasing the coupling inductance $L_{\mathrm{AF}}$. However, due to parameter sensitivity and non-ideal converter characteristics, the control accuracy is likely to decrease for a reduction of the 
coupling inductance. In order to overcome this disadvantage, (7) reveals important advantages of the exception $L_{\mathrm{AF}}=0$. Omission of the active filter coupling inductance at the same time reduces the parameter sensitivity and entails the lowest achievable active filter voltage demand amounting to the rated grid voltage, leading to a voltage-source output characteristic of the hybrid converter. The derived voltage-source hybrid converter is introduced and investigated in the following.

\section{PARt II: Voltage-Source Hybrid Converter}

In comparison to the current-source hybrid converter, the omission of the active filter coupling inductor $L_{\mathrm{AF}}$ (low current rating) necessitates an additional grid-side coupling inductor $L_{\mathrm{F}, \mathrm{G}}$ (high current rating), as can be seen in Fig. 8 . Due to the low rated power, the active filter can be designed with a high number of voltage levels and operated at a high switching frequency, allowing the use of a low grid-side inductance.

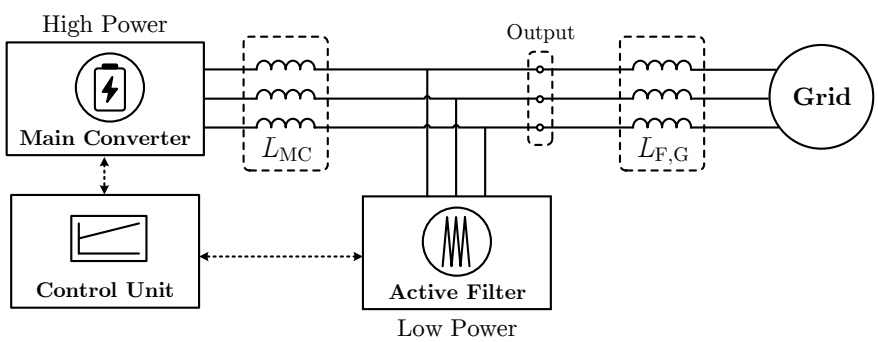

Fig. 8. Proposed grid-connected voltage-source hybrid converter topology

\section{A. Converter Topology and Analysis}

In order to ensure comparability to the current-source hybrid converter, the topologies of the main converter and active filter in Fig. 9 remain a 2L-VSC and CHB STATCOM, respectively. By the direct parallel connection to the output, the active filter provides the output voltage of the hybrid converter and thereby determines the output current. Consequently, the voltage-source hybrid converter possesses a multilevel output voltage and can be operated in any VSC application. Compared to conventional multilevel converters, it is expected that the hybrid converter allows distinct increased power densities due to the high power conventional main converter and the low power output mulitlevel converter. In contrast to the Modular Multilevel Converter (MMC), the low frequency operation down to output frequency zero is feasible without additional control effort and thus the frequency independent operation constitutes another important advantage of the hybrid converter.

Kirchhoff's Current Law at the converter output yields

$$
i_{\mathrm{G}}=i_{\mathrm{MC}}-i_{\mathrm{AF}},
$$

leading to an inherent compensation of the main converters switching frequency current ripple, since the output current $i_{\mathrm{G}}$ is solely dependent on the output voltage of the active filter. However, it has to be noted that the main converter control must ensure the proper operation of the total converter system by preventing operating points, in which the active filter draws not only the switching frequency current ripple but also fundamental current.

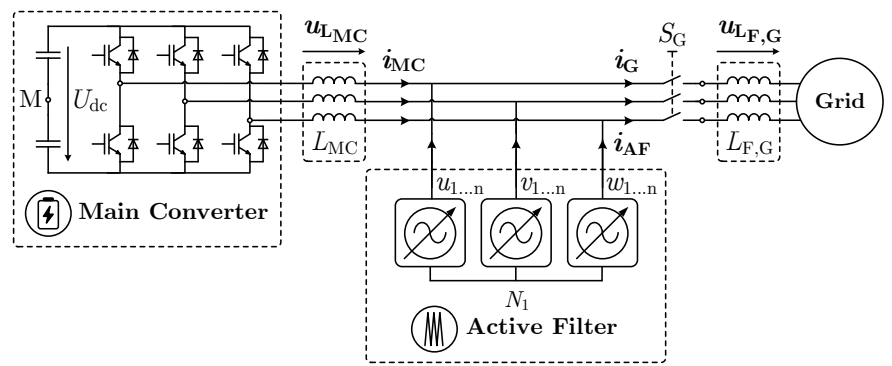

Fig. 9. Proposed voltage-source hybrid converter composed of a $2 \mathrm{~L}-\mathrm{VSC}$ as main converter and a CHB STATCOM in star configuration as active filter

\section{B. Converter Control}

The control scheme of the voltage-source hybrid converter is depicted in Fig. 10. In comparison to the control scheme of the current-source hybrid converter introduced in section II-B, the active filter control is independent of the main converter control. Since the active filter directly provides the hybrid converter output voltage, it performs the grid-side control to allow the accurate injection of the reference current $\underline{i}_{\mathrm{G}, \mathrm{dq}}^{*}$. Therefore a conventional grid-side fundamental frequency control is applied, using a voltage feed-forward in order to improve the dynamic behavior. The feed-forward voltage is given by the grid voltage provided by a phase-locked loop (PLL) and the load voltage drop caused by the injected current, resulting in

$$
\underline{u}_{\mathrm{L}, \mathrm{G}, \mathrm{dq}}=\underline{u}_{\mathrm{G}, \mathrm{dq}}+\underline{i}_{\mathrm{G}, \mathrm{dq}}^{*} \underline{Z}_{\mathrm{F}, \mathrm{G}}
$$

with the grid-side coupling impedance

$$
\underline{Z}_{\mathrm{F}, \mathrm{G}}=R_{\mathrm{F}, \mathrm{G}}+j \omega_{\mathrm{G}} L_{\mathrm{F}, \mathrm{G}} .
$$

The main converter takes on the internal control of the hybrid converter, ensuring that the entire active power exchanged with the grid is provided by the high power $2 \mathrm{~L}-\mathrm{VSC}$. Thus, the CHB STATCOM merely provides the harmonic reactive power of the compensated ripple current and can be designed for a low power rating. Due to the fundamental frequency control scheme, this is achieved by using the measured injected grid current as the reference value and the measured main converter current as the actual value. With respect to the main converter current composition in (4) and Kichhoff's Current Law in (8), this leads to the intended active filter current

$$
i_{\mathrm{AF}}=\sum \nu i_{\mathrm{MC}} .
$$

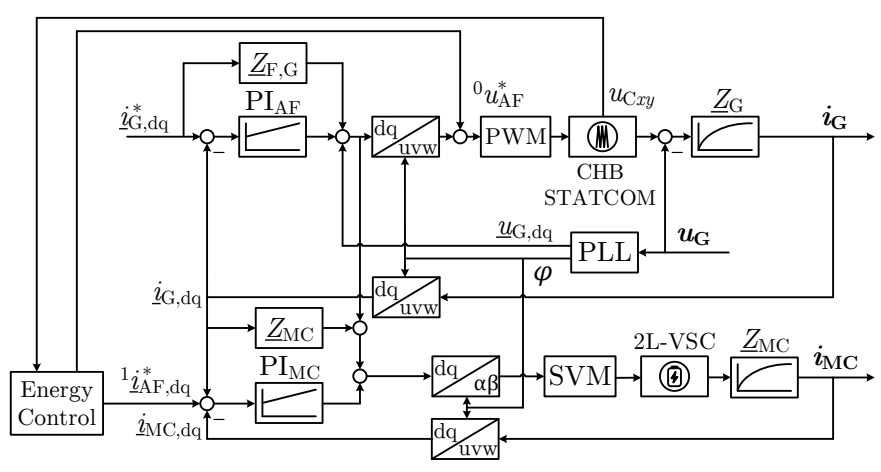

Fig. 10. Control scheme of the proposed voltage-source hybrid converter 
Moreover, since there is no DC-link supply of the CHB STATCOM, an energy control is applied for the voltage-source hybrid converter. In order to ensure a constant voltage level overall the three phases as well as among each phase, the energy control uses the measured CHB STATCOM cell voltages $u_{\mathrm{C} x y}$ (with $x=\{u, v, w\}$ and $y=\{k \in \mathbb{N} \mid 1 \leq k \leq 12\}$ ). Following the energy balancing strategies introduced in [8], the total three-phase energy as well as the energy per phase of the CHB STATCOM is balanced using a low fundamental current ${ }^{1} \underline{\mathrm{i}}_{\mathrm{AF}, \mathrm{dq}}^{*}$ and the common mode voltage ${ }^{0} u_{\mathrm{AF}}^{*}$.

\section{Simulation Results}

Simulation results are carried out according to the simulation parameters of the current-source hybrid converter in Tab. I, while the active filter inductance is used as an additional gridside inductance $L_{\mathrm{AF}}=L_{\mathrm{F}, \mathrm{G}}=200 \mu \mathrm{H}$. In comparison to the simulation results carried out for the current-source hybrid converter, the voltage-source hybrid converter operation in Fig. 11 is performed with precharging as well as energy balancing of the CHB STATCOM cell capacitors during operation by using the control scheme introduced in section III-B. Using precharging resistors $R_{\mathrm{p}}=5 \Omega$ in series connection to the CHB STATCOM, the cell capacitors with $C_{\mathrm{c}, \mathrm{AF}}=6 \mathrm{mF}$ are initally charged to the reference voltage $u_{\mathrm{c}, \mathrm{AF}}=35 \mathrm{~V}$. Since the hybrid converter is not yet connected to the grid, the charging current is provided by the main converter. At $t=30 \mathrm{~ms}$ the precharging resistors are bypassed and the CHB STATCOM is directly connected to the hybrid converter output, leading to a reduced damping of the main converter current ripple and an increased current load of the active filter. After synchronization, performed by a phase-locked loop, the hybrid converter is connected to the grid at $t=60 \mathrm{~ms}$. The reference current is increased to $\hat{i}_{\mathrm{G}}^{*}=200 \mathrm{~A}$ and $\hat{i}_{\mathrm{G}}^{*}=400 \mathrm{~A}$ at $t=100 \mathrm{~ms}$ and $t=150 \mathrm{~ms}$, respectively. High control dynamics are achieved for the reference current step changes, while the active filter load is certain limited to the main converter current ripple. However, during steady state operation at a high reference current, a low fundamental balancing current ${ }^{1} \hat{i}_{\mathrm{AF}}$, resulting from unsymmetrical current ripples in the three phases of the main converter, ensures an accurate energy balancing of the active filter. The injected grid current reveals an excellent harmonic performance of the hybrid converter. During the injection of $\hat{i}_{\mathrm{G}}=200 \mathrm{~A}$ the main converter current distortion is $\mathrm{THD}_{\mathrm{MC}}=11.9 \%$ and the mitigation of the switching frequency current ripple by the active filter causes a grid current distortion of only $\mathrm{THD}_{\mathrm{G}}=0.3 \%$. Due to the additionally provided transient balancing current and the non-sinusoidal output voltage, the main converter current distortion is slightly increased compared to the current-source hybrid converter. Nevertheless, the distortion of the active filter multilevel voltage is low and the grid current distortion achieved by the voltage-source hybrid converter is further reduced by $0.1 \%$. In consequence of the increased fundamental component, the current distortions decrease to $\mathrm{THD}_{\mathrm{MC}}=5.1 \%$ and $\mathrm{THD}_{\mathrm{G}}=0.2 \%$ for $\hat{i}_{\mathrm{G}}=400 \mathrm{~A}$.
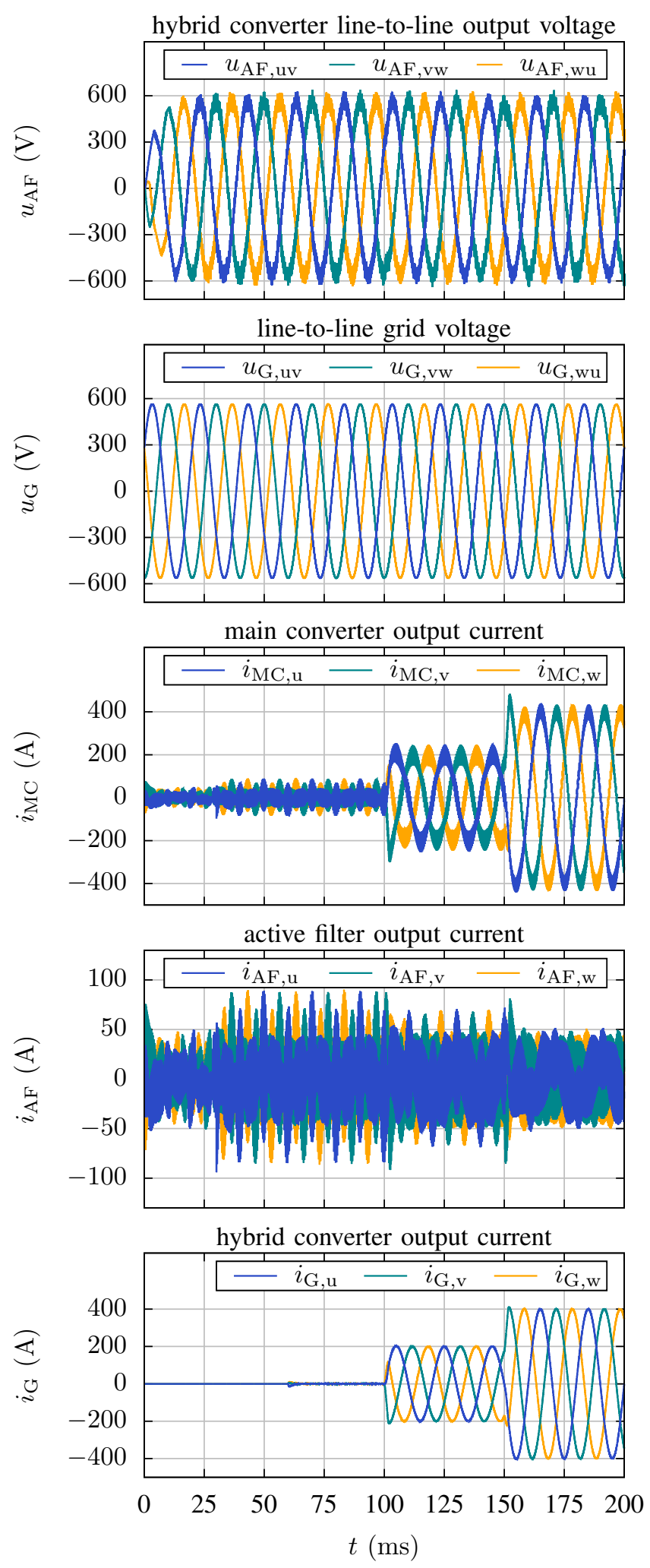

Fig. 11. Simulation results of the voltage-source hybrid converter

It can be seen that the active filter current is effectively limited and stays below $\hat{i}_{\mathrm{AF}}=85 \mathrm{~A}$ during steady state and dynamic operation. The maximum switching frequency current ripple 
of the main converter $\sum \nu \boldsymbol{i}_{\mathrm{MC}}$ has an apparent power of $\sum^{\nu} S_{\mathrm{MC}}=14 \mathrm{kVA}$. As a result of a maximum required balancing current of ${ }^{1} \hat{i}_{\mathrm{AF}}=10 \mathrm{~A}$, leading to a balancing power of ${ }^{1} S_{\mathrm{AF}}=5 \mathrm{kVA}$, the active filter in this application is to be designed for a maximum rated power of

$$
S_{\mathrm{AF}}={ }^{\sum \nu} S_{\mathrm{MC}}+{ }^{1} S_{\mathrm{AF}}=19 \mathrm{kVA} .
$$

Assuming main converter current ratings of $\hat{i}_{\mathrm{MC}}=200 \mathrm{~A}$ and $\hat{i}_{\mathrm{MC}}=400 \mathrm{~A}$, leading to power ratings of $S_{\mathrm{MC}}=98 \mathrm{kVA}$ and $S_{\mathrm{MC}}=196 \mathrm{kVA}$, the hybrid converter power ratios given by

$$
s=\frac{S_{\mathrm{AF}}}{S_{\mathrm{MC}}}
$$

are $s=19.4 \%$ and $s=9.7 \%$, respectively. This is clearly illustrating the high potential of the voltage-source hybrid converter regarding power density, cost and efficiency competitiveness to both conventional grid-connected converters as well as multilevel converters for any VSC application.

\section{Comparison to LCL-filter based converter systems}

The investigation of the voltage-source hybrid converter was carried out for a grid-side inductance $L_{\mathrm{F}, \mathrm{G}}=200 \mu \mathrm{H}$ in order to ensure comparability to the current-source hybrid converter. The hybrid converter system in Fig. 9 attains a superior harmonic output performance with grid current total harmonic distortions of $0.3 \%$ and $0.2 \%$ for $\hat{i}_{\mathrm{G}}=200 \mathrm{~A}$ and $\hat{i}_{\mathrm{G}}=400 \mathrm{~A}$, respectively. However, comparing the competitiveness to conventional grid-connected converter systems with output LCL-filters requires the consideration of common harmonic limits, which are likely to still be met when reducing the grid-side inductance to values that are e.g. in the range of grid-side transformer leakage inductances. To meet the harmonic limits given by IEEE Standard 519 with a $100 \mathrm{kVA}$ converter system, the total required filter inductance

$$
L_{\mathrm{F}(\mathrm{HC})}=L_{\mathrm{MC}}+L_{\mathrm{F}, \mathrm{G}}
$$

of the voltage-source hybrid converter and a LCL-filter based Two-Level converter, relating to a reference LCL-filter design $\left(L_{\mathrm{F}(\mathrm{LCL}, \mathrm{ref})}=L_{\mathrm{F}, \mathrm{C}}+L_{\mathrm{F}, \mathrm{G}}\right)$ according to [10], are investigated. Therefore, the total filter inductances of the converter systems are minimized with respect to the switching frequency $f_{\mathrm{sw}}$ and the hybrid converter power ratio $s$ in (13). The results shown in Fig. 12 clearly confirm the expected high potential of the voltage-source hybrid converter for the reduction in passive component cost. Even for very low power ratios of $s \leq 10 \%$, considerable reduced total inductances of $40 \%$ and $50 \%$ can be achieved for switching frequencies of $f_{\mathrm{sw}} \leq 8 \mathrm{kHz}$ and $f_{\mathrm{sw}} \leq 6 \mathrm{kHz}$, respectively, compared to the inductance requirement of the reference LCL-filter design. Furthermore, an inversely proportional relation of the power ratio $s$ and the relative total inductance $L_{\mathrm{F}(\mathrm{HC})} / L_{\mathrm{F} \text { (LCL,ref) }}$ can be noted in Fig. 12. This is caused by the elementary reactive power characteristics of the filter inductance. The fundamental reactive power is proportional to the fundamental current, determined by the converters power rating, and to the filter inductance. Neglecting the low fundamental current of both the active
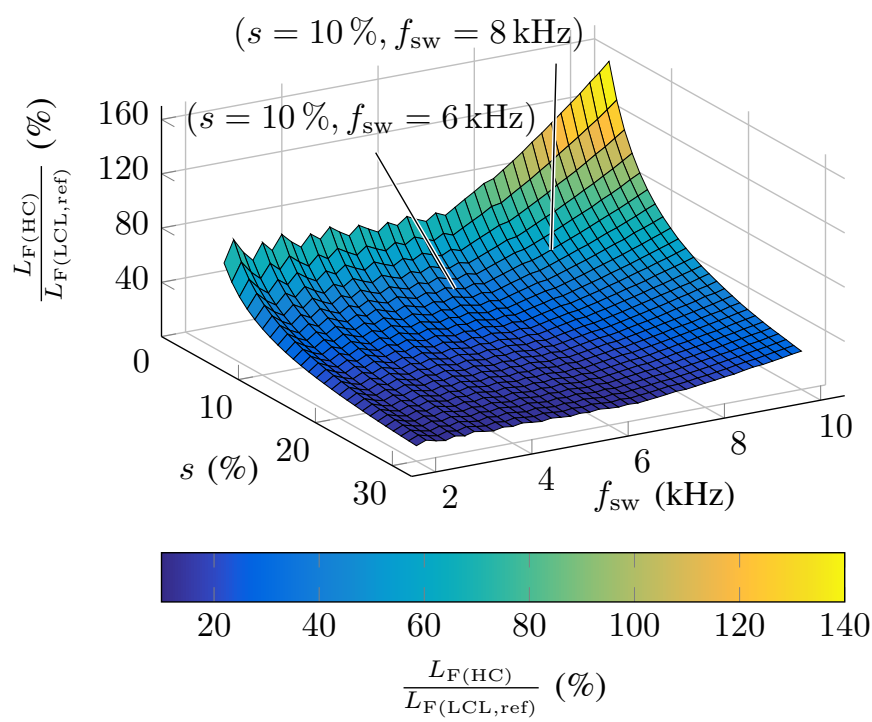

Fig. 12. Comparison of the total filter inductance demand of the voltage-source hybrid converter and a LCL-filter based $2 \mathrm{~L}-\mathrm{VSC}$ as a function of the power ratio $s$ and the switching frequency $f_{\mathrm{sw}}$ for a $100 \mathrm{kVA}$ system

filter of the hybrid converter and the filter capacitors of the LCL-filter based system, the single phase fundamental reactive power of the filter inductance results in

$$
{ }^{1} Q_{\mathrm{L}_{\mathrm{F}}}=\left|{ }^{1} \underline{i}_{\mathrm{MC}}\right|^{2} \omega_{\mathrm{G}}\left(L_{\mathrm{MC}}+L_{\mathrm{F}, \mathrm{G}}\right)
$$

with the fundamental current of the main converter

$$
{ }^{1} \underline{i}_{\mathrm{MC}}=\frac{{ }^{1} \underline{u}_{\mathrm{MC}}-{ }^{1} \underline{u}_{\mathrm{G}}}{j \omega_{\mathrm{G}}\left(L_{\mathrm{MC}}+L_{\mathrm{F}, \mathrm{G}}\right)} .
$$

Fourier analysis of the main converter, active filter and grid voltage gives the harmonic reactive power

$$
\begin{aligned}
& \sum{ }^{\nu} Q_{\mathrm{L}_{\mathrm{F}}}={ }^{\sum \nu} Q_{\mathrm{L}_{\mathrm{MC}}}+{ }^{\sum \nu} Q_{\mathrm{L}_{\mathrm{F}, \mathrm{G}}} \\
= & \sum_{\nu=2}^{\infty}\left(\frac{\left|{ }^{\nu} \underline{u}_{\mathrm{MC}}-{ }^{\nu} \underline{u}_{\mathrm{AF}}\right|^{2}}{\nu \omega_{\mathrm{G}} L_{\mathrm{MC}}}+\frac{\left|{ }^{\nu} \underline{u}_{\mathrm{AF}}-{ }^{\nu} \underline{u}_{\mathrm{G}}\right|^{2}}{\nu \omega_{\mathrm{G}} L_{\mathrm{F}, \mathrm{G}}}\right)
\end{aligned}
$$

whereas the active filter voltage has to be replaced by the filter capacitor voltage for the LCL-filter based system. It can be assumed that the entire switching frequency current ripple is filtered by the active filter of the hybrid converter system as well as by the filter capacitors of the LCL-filter based converter, thereby making $\sum^{\nu} Q_{\mathrm{L}_{\mathrm{F}, \mathrm{G}}}$ negligible. Fig. 13 shows the fundamental, the harmonic and the total reactive power $Q_{\mathrm{L}_{\mathrm{F}}}={ }^{1} Q_{\mathrm{L}_{\mathrm{F}}}+{ }^{\sum \nu} Q_{\mathrm{L}_{\mathrm{F}}}$ for a rated power of $100 \mathrm{kVA}$ and a switching frequency of $3 \mathrm{kHz}$. A global minimum of the inductive reactive power occurs at a very low filter inductance of $L_{\mathrm{F}} \approx 0.03 \mathrm{mH}$. Fig. 14 shows the total inductive reactive power as a function of the total filter inductance with a larger scale. As expected, with a reduction of the filter inductance the required active filter rated power increases in order to meet the harmonic limits, since the switching frequency voltage ripple of the main converter voltage $\sum \nu \boldsymbol{u}_{\mathrm{MC}}$ leads to an increased

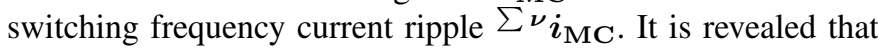
LCL-filter based converter systems require a distinct increased 


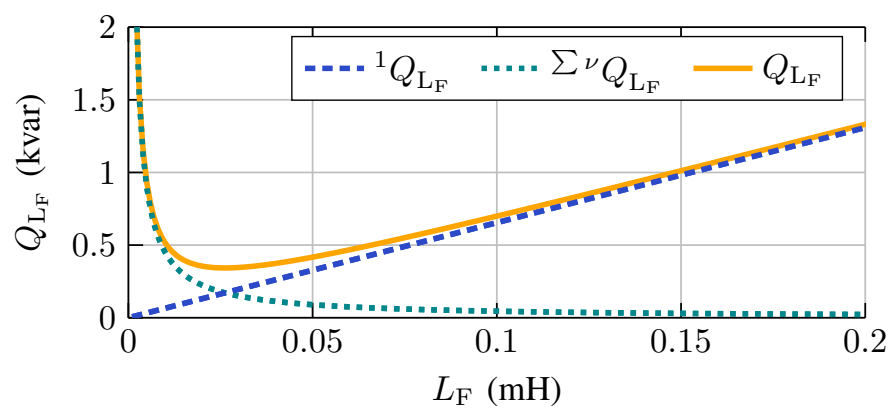

Fig. 13. Reactive power composition as a function of the total filter inductance

filter inductance, since not only the reference LCL-filter design surpasses low hybrid converter power ratios, but the for LCLfilter design commonly applied capacitive reactive power limit of $q_{\mathrm{C}, \mathrm{LCL}} \leq 5 \%$ as well surpasses a power ratio of $s=5 \%$.

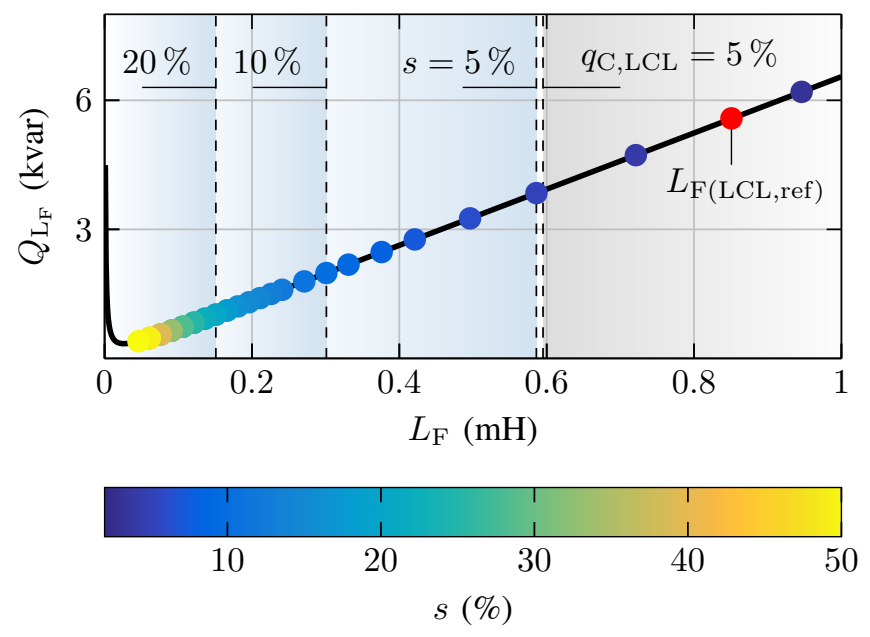

Fig. 14. Inductance demand and corresponding inductive reactive power of the voltage-source hybrid converter and the reference LCL-filter based 2L-VSC

\section{CONCLUSION}

A novel hybrid converter topology composed of a high power main converter with an integrated Multilevel-based active filter using a coupled control scheme is proposed in this contribution. In a first step a conventional active filter coupling network is considered, leading to current-source output characteristics of the hybrid converter. Based on the currentsource converter system analysis a coupled control scheme is introduced for the combination of a Two-Level converter and a CHB STATCOM, allowing the immediate mitigation of the switching frequency current ripple. Simulation results reveal a excellent harmonic performance of the hybrid converter under reasonable grid conditions, reducing the total harmonic distortion of the output current to below $0.5 \%$. As the active filter solely compensates the switching frequency current ripple of the main converter, it requires merely a fractional amount of the main converter power rating. Due to the main disadvantages for practical implementation given by the increased voltage demand of the active filter as well as the expected parameter sensitivity, a voltage-source hybrid converter is derived by eliminating the active filter coupling inductance, leading to a reduced active filter voltage demand amounting to the grid voltage level. The developed control scheme, including an energy balancing control of the active filter, shows excellent dynamic and harmonic performance. A comparison to LCLfilter based grid-connected converter systems reveals a high potential for reduction of inductive filter component cost, allowing a reduction in inductance of up to $50 \%$ even for very low active filter to main converter power rating ratios of $10 \%$. Moreover, due to the voltage-source output characteristics, the hybrid converter is suited for any VSC application, achieving superior power density compared to conventional multilevel converters. Therefore, the introduced hybrid converter concept is promising for increasing the efficiency and power density as well as decreasing the costs of low- and medium voltage converters in a wide field of applications.

\section{REFERENCES}

[1] S. N. Kalaschnikow. "High efficient low harmonic drives with integrated active filters". In: 2013 15th European Conference on Power Electronics and Applications (EPE). IEEE, Sept. 2013.

[2] R. Hou et al. "A Primary Full-Integrated Active Filter Auxiliary Power Module in Electrified Vehicles With Single-Phase Onboard Chargers". In: IEEE Transactions on Power Electronics 32.11 (Nov. 2017), pp. 8393-8405.

[3] A. K. Jain et al. "Wound Rotor Induction Generator With Sensorless Control and Integrated Active Filter for Feeding Nonlinear Loads in a Stand-Alone Grid". In: IEEE Transactions on Industrial Electronics 55.1 (Jan. 2008), pp. 218-228.

[4] E. Behrouzian et al. "Impact of Switching Harmonics on Capacitor Cells Balancing in Phase-Shifted PWM-Based Cascaded H-Bridge STATCOM". In: IEEE Transactions on Power Electronics 32.1 (Jan. 2017), pp. 815-824.

[5] Y. Zhang et al. "Impedance modeling and analysis of MMC in single-star configuration". In: 2017 IEEE 18th Workshop on Control and Modeling for Power Electronics (COMPEL). IEEE, July 2017.

[6] M. Pereira et al. "SVC PLUS: An MMC STATCOM for network and grid access applications". In: 2011 IEEE Trondheim PowerTech. IEEE, June 2011.

[7] ABB AB. SVC Light: For electrical transmission grids. Brochure ABB FACTS, Vasteras, Sweden, 2014.

[8] F. Kammerer et al. "Energy balancing of the Modular Multilevel Matrix Converter based on a new transformed arm power analysis". In: 2014 16th European Conference on Power Electronics and Applications. IEEE, Aug. 2014.

[9] T. Geyer et al. "Model Predictive Direct Torque Control-Part I: Concept, Algorithm, and Analysis". In: IEEE Transactions on Industrial Electronics 56.6 (June 2009), pp. 1894-1905.

[10] K. Jalili et al. "Design of LCL Filters of Active-FrontEnd Two-Level Voltage-Source Converters". In: IEEE Transactions on Industrial Electronics 56.5 (May 2009), pp. 1674-1689. 